\title{
Numerical Simulation of High Frequency Wave Coupling within a Hall Thruster
}

\author{
A. Knoll ${ }^{1}$, N. Gascon ${ }^{2}$, and M. Cappelli ${ }^{3}$ \\ Stanford University, Stanford, CA, 94305
}

\begin{abstract}
[Abstract] A 2-dimensional Hall thruster simulation has been developed in the axialazimuthal coordinate plane. The goal of this simulation is to numerically model high frequency plasma waves within the discharge channel of the Hall thruster, and study the contribution of these waves to the time-averaged axial electron drift. This model uses a continuum (fluid) representation for both the electrons and ions. In order to simulate oscillations in the electron field it was necessary to model the electrons dynamically, as opposed to assuming a steady state solution at each time step. The electron momentum equations also include electron inertia terms that are normally neglected in typical Hall thruster models. These inertia terms provide a wave coupling mechanism between axially and azimuthally propagating waves. This numerical model was able to reproduce two dominant high frequency plasma oscillations in the Hall thruster: a $74 \mathrm{MHz}$ KelvinHelmholtz type shearing instability, and a $7 \mathrm{MHz}$ oscillation in the plasma density that has also been observed experimentally. The simulation was successful at predicting the axial electron drift in good agreement with experiment. The results of this study suggest that the plasma oscillations play a dominant role in the electron transport process. In particular, contributions to the electron transport resulting from perturbations in the azimuthal electron velocity were found to be greater than $300 \%$ of classical collisional transport.
\end{abstract}

\section{Nomenclature}

$B_{r} \quad=$ radial magnetic flux density

$E_{z} \quad=$ axial electric field

$E_{\theta} \quad=$ azimuthal electric field

e

$=$ electron charge

$=$ current density

$=$ Boltzmann constant

$=$ electron mass

$=$ ion mass

$=$ number density

$=$ mean radius of the acceleration channel

$=$ electron temperature

$=$ time

$=$ axial electron velocity

$=$ azimuthal electron velocity

$=$ axial ion velocity

$=$ azimuthal ion velocity

$=$ axial position

$=$ azimuthal position

$=$ electron mobility

$=$ electon-neutral collision frequency

$=$ mean time between electron-neutral collisions

$=$ plasma potential

\footnotetext{
${ }^{1}$ Research Assistant, Mechanical Engineering: Thermosciences Division, Building 520, AIAA Student

${ }^{2}$ Postdoctoral Fellow, Mechanical Engineering: Thermosciences Division, Building 520, AIAA Member

${ }^{3}$ Professor, Mechanical Engineering: Thermosciences Division, Building 520, AIAA Member
} 


\section{Introduction}

$\mathrm{T}$ his paper describes a high fidelity simulation of a Hall thruster that has been formulated in the axial-azimuthal coordinate plane. The goal of this numerical model is to capture the high frequency plasma oscillations observed experimentally in Hall thrusters. The oscillations under consideration in this study are between $1 \mathrm{MHz}$ and $100 \mathrm{MHz}$. Of particular interest is the contribution that these oscillations have on the time-averaged axial electron transport.

One of the main challenges in the field of Hall thruster research today is the anomalously high axial electron drift compared with classical predictions. A number of studies have suggested that the anomalous electron drift is related to high frequency plasma oscillations. ${ }^{1,2}$ However, no theory has emerged that can quantify this phenomena over the various operating regimes of the Hall thruster. Typical practice in the simulation of Hall thrusters is to introduce an experimentally motivated anomalous transport parameter or a Bohm type mobility. However, this parameter is heavily dependent on the operating regime of the thruster, and ultimately limits the usefulness of these simulations. The goal of this study is to numerically capture various high frequency oscillations and determine the contribution they have to the time-averaged axial electron drift.

The traditional approach to studying wave modes in a Hall Thruster involves linear analysis of small amplitude perturbations. Although this approach is quite successful at describing a multitude of wave modes harbored by the plasma, it reveals little about which of these possible modes is dominant in the thruster. Also, this simplified analysis is unable to predict how energy carried by one wave can excite other wave modes entirely. This is an inherently non-linear process that is lost when making the small amplitude assumption. This study seeks to include the non-linear terms and study high frequency wave coupling within the Hall Thruster.

This simulation uses a continuum (fluid) representation for both the electrons, ions, plasma potential, and plasma density. Several terms have been included in the governing equations that are normally neglected in traditional Hall thruster models. These are the transient components of the electron velocity and the convective terms in the momentum equation for both the electrons and ions. These terms have been included in this study to allow high frequency instabilities to develop and interact in electron and ion fields.

Hall thruster simulations are typically formulated in the radial-axial coordinates of the thruster as illustrated in Fig. 1. The alternative axial-azimuthal computational domain (see Fig. 2) was selected so that both azimuthal and axial plasma oscillations could be resolved. The computational domain includes the full azimuth of the thruster, and a $1 \mathrm{~cm}$ axial slice, positioned in the peak magnetic field region of the thruster. The location of the computational domain corresponds to a region of the thruster where plasma oscillations are expected to be the highest. This is also the region where the most complex behavior in the axial electron transport are typically observed.

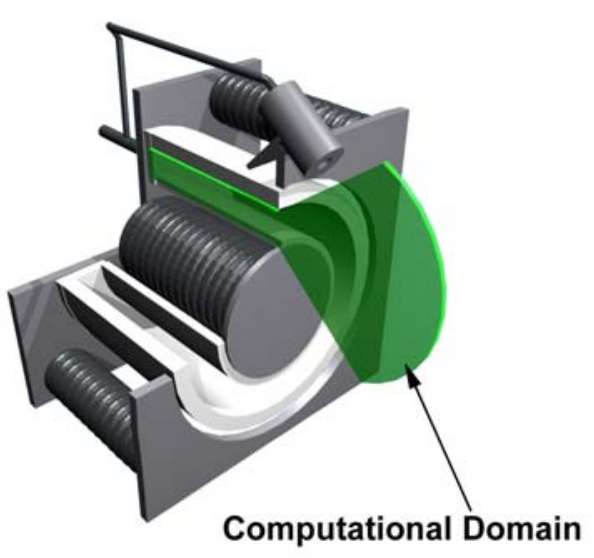

Figure 1. Radial-Axial Computational Domain.

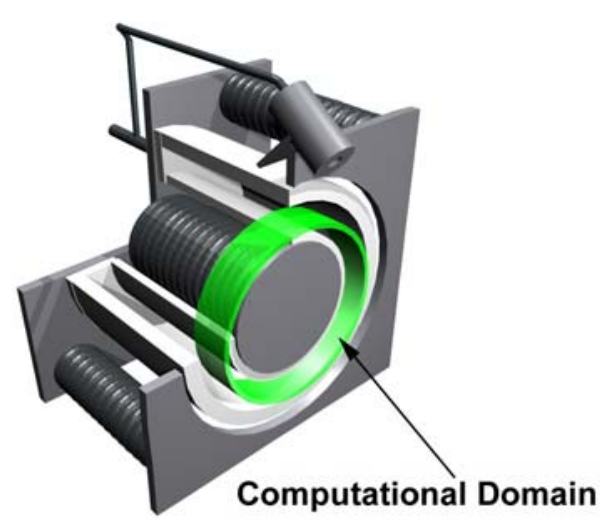

Figure 2. Axial-Azimuthal Computational Domain. 


\section{Governing Equations}

The numerical model used in this study is 2dimensional in the axial $(\mathrm{z})$ - azimuthal $(\theta)$ plane of the thruster. This coordinate system is illustrated in Fig. 3. The system consists of 6 unknowns: two components of electron velocity, two components of ion velocity, plasma density, and plasma potential. The electron temperature is taken to be constant, with an axially varying value supplied by experimental measurements. The constant temperature approximation is likely justified due to the high frequency nature of the oscillations considered in this study. However, this condition limits the simulation to operating regimes where experimental electron temperature data is available. Future work calls for including the electron energy equation in order to selfconsistently predict Hall thruster performance parameters.

Each of the six plasma parameters is modeled as a dynamic fluid continuum. Six equations are required to specify the system. The first two equations are the axial

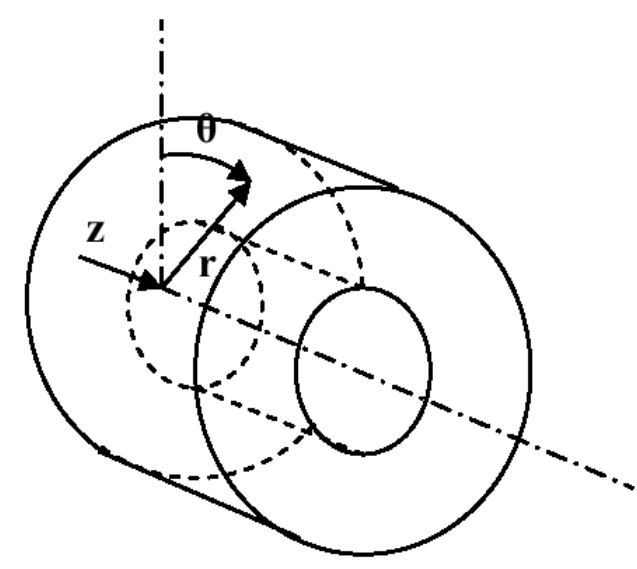
and azimuthal components of the electron momentum equation given by

$$
\begin{aligned}
& n m_{e} \frac{\partial u_{e z}}{\partial t}+n m_{e} u_{e z} \frac{\partial u_{e z}}{\partial z}+\frac{n m_{e} u_{e \theta}}{R} \frac{\partial u_{e z}}{\partial \theta}=n e\left(\frac{\partial \phi}{\partial z}-u_{e \theta} B_{r}\right)-m_{e} u_{e z} n v_{e N}-k \frac{\partial\left(n T_{e}\right)}{\partial z} \text {, and } \\
& n m_{e} \frac{\partial u_{e \theta}}{\partial t}+n m_{e} u_{e z} \frac{\partial u_{e \theta}}{\partial z}+\frac{n m_{e} u_{e \theta}}{R} \frac{\partial u_{e \theta}}{\partial \theta}=n e\left(\frac{1}{R} \frac{\partial \phi}{\partial \theta}+u_{e z} B_{r}\right)-m_{e} u_{e \theta} n v_{e N}-\frac{k}{R} \frac{\partial\left(n T_{e}\right)}{\partial \theta} .
\end{aligned}
$$

Several terms have been retained in these equations that are normally discarded for simplicity. The left hand side of these equations include the transient behavior and the momentum contribution of the electrons that are traditionally neglected. Also, the coordinate system considered in this study allows for electric fields to develop in the azimuthal direction. These terms have been included in this analysis in order to establish their significance to the axial electron transport process. Also, these terms are necessary to model the behavior of plasma oscillations that involve the electron field.

The next two equations are the ion momentum equations in the axial and azimuthal directions given by

$$
\begin{aligned}
& n m_{i} \frac{\partial u_{i z}}{\partial t}+n m_{i} u_{i z} \frac{\partial u_{i z}}{\partial z}+\frac{n m_{i} u_{i \theta}}{R} \frac{\partial u_{i z}}{\partial \theta}=-n e \frac{\partial \phi}{\partial z}-k \frac{\partial\left(n T_{i}\right)}{\partial z}, \text { and } \\
& n m_{i} \frac{\partial u_{i \theta}}{\partial t}+n m_{i} u_{i z} \frac{\partial u_{i \theta}}{\partial z}+\frac{n m_{i} u_{i \theta}}{R} \frac{\partial u_{i \theta}}{\partial \theta}=\frac{-n e}{R} \frac{\partial \phi}{\partial \theta}-\frac{k}{R} \frac{\partial\left(n T_{i}\right)}{\partial \theta} .
\end{aligned}
$$

As in the case of the electron momentum equations, the non-linear ion momentum terms have been retained in these equations. Although their net contributions to the ion velocity may be small, they provide the only wave coupling mechanism between axial and azimuthal disturbances in the ion field.

The fifth equation is a statement of charge conservation, which can be expressed as

$$
\frac{-\partial\left(n u_{e z}\right)}{\partial z}+\frac{\partial\left(n u_{i z}\right)}{\partial z}-\frac{1}{R} \frac{\partial\left(n u_{e \theta}\right)}{\partial \theta}+\frac{1}{R} \frac{\partial\left(n u_{i \theta}\right)}{\partial \theta}=0 \text {. }
$$

Finally, an expression for the conservation of mass completes the system. This is given by 


$$
\frac{\partial n}{\partial t}+\frac{\partial\left(n u_{i z}\right)}{\partial z}+\frac{1}{R} \frac{\partial\left(n u_{i \theta}\right)}{\partial \theta}=0
$$

In all the above equations ionization has been neglected, and quasi-neutrality has been assumed.

\section{Boundary Conditions}

The computational domain used for this analysis generally requires two boundary conditions for each of the six plasma parameters specified at the upstream and downstream planes, plus an assumption of periodicity in the azimuthal direction. For subsequent discussion, the boundary closest to the anode will be termed boundary "A", and the boundary closest to the cathode will be boundary "B".

The boundary conditions are established for the electrons by assuming they enter the computational domain from the cathode normal to boundary "B". Their velocity at this point can be determined by the net Hall thruster current, which is taken to be constant. A Neumann type condition is specified at boundary "A" for the axial electron velocity by solving the conservation of charge and mass equations and neglecting temporal variations in plasma density. Finally, we arbitrarily set the Neumann condition on the azimuthal electron velocity at boundary "A" to be zero, as the solution appears to be only weakly dependent on this condition. The axial electron velocity boundary conditions can then be written

$$
\begin{gathered}
{\left[\frac{\partial\left(n u_{e z}\right)}{\partial z}\right]_{A}=\frac{-1}{R} \frac{\partial\left(n u_{e \theta}\right)}{\partial \theta}, \text { and }} \\
{\left[u_{e z}\right]_{B}=u_{i z}-\frac{J}{n e} .}
\end{gathered}
$$

The azimuthal electron velocity boundary conditions are

$$
\begin{gathered}
{\left[\frac{\partial u_{e \theta}}{\partial z}\right]_{A}=0 \text {, and }} \\
{\left[u_{e \theta}\right]_{B}=0 .}
\end{gathered}
$$

The conditions for the plasma potential at boundary "A" are taken to be azimuthally constant at a value provided by experimental measurements. For this study it is set at $140 \mathrm{~V}$. A Neumann type condition on the plasma potential at boundary " $\mathrm{B}$ " is derived from the ion momentum equation. The ion momentum equation is used in favor of the electron momentum because the transient terms in the ion field are small and lead to a more stable boundary less prone to numerical instabilities. The plasma boundary conditions can be expressed by

$$
\begin{gathered}
\phi_{A}=140[\mathrm{~V}], \text { and } \\
{\left[\frac{\partial \phi}{\partial z}\right]_{B}=\frac{-m_{i} u_{i z}}{e} \frac{\partial u_{i z}}{\partial z}-\frac{m_{i} u_{i \theta}}{e R} \frac{\partial u_{i z}}{\partial \theta}}
\end{gathered}
$$

The axial ion velocity in specified at $10 \mathrm{~km} / \mathrm{s}$ entering the computational domain normal to boundary "A". A Neumann condition on the outgoing ions through boundary " $\mathrm{B}$ " comes from the conservation of mass equation. For the ions we have

$$
\begin{gathered}
{\left[u_{i z}\right]_{A}=10000[\mathrm{~m} / \mathrm{s}], \text { and }} \\
{\left[\frac{\partial\left(n u_{i z}\right)}{\partial z}\right]_{B}=\frac{-1}{R} \frac{\partial\left(n u_{i \theta}\right)}{\partial \theta} .}
\end{gathered}
$$


Finally, the plasma density is specified from experimental measurements at boundary "A" by

$$
n_{A}=1 \times 10^{17}\left[\mathrm{~m}^{-3}\right] \text {. }
$$

No condition is needed at boundary " $\mathrm{B}$ " for the plasma density because the conservation of mass equation, which is used to solve for the plasma density, is a first order advection equation.

\section{Numerical Technique}

The following procedure is used at each iteration step to advance the model in time. First, the axial and azimuthal electron momentum equations are combined to produce a Poisson type equation for the plasma potential. A finite element method is then used to solve this equation. Second, the electron field is advanced in time using the electron momentum equations. These equations are solved using the trapezoidal time step method in conjunction with a second-order finite differencing scheme for the spatial derivatives. Third, the ion field is advanced in time using the ion momentum equations. The same numerical technique is employed for the ions as with the electrons. Fourth, the conservation of mass equation is used to update the plasma density field. Finally, the system boundary conditions are applied as described above. This procedure is shown schematically in Fig 4.

The simulation domain was discretized into $625 \times 25$ cells in the azimuthal and axial directions respectively. The time step used for the simulation was $0.1 \mathrm{~ns}$. This seemingly restrictive time step was needed because of the transient terms included in the electron momentum

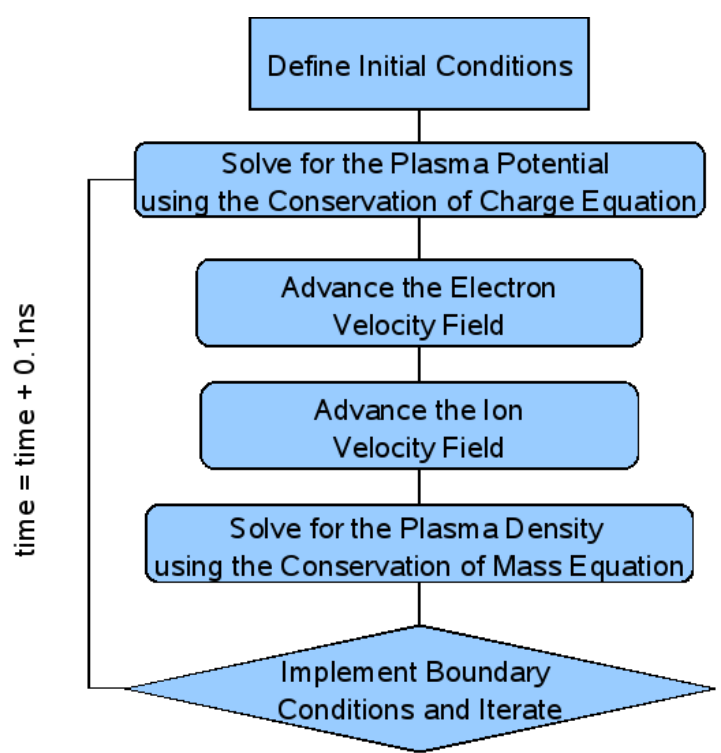

Figure 4. Simulation Flowchart. equation.

The simulation takes approximately $2 \frac{1}{2}$ days to solve for $1 \mu$ s of simulated time. This computation is currently being performed on a dual processor Opteron 270 computer running on the SuSE Linux-64 operating system.

A visualization of the simulation output is shown in Fig 5. This image clearly shows the development of a wave in the axial electron velocity. The magnitude of these oscillations can be up to an order of magnitude greater than the mean value. Animations of this wave reveal that it is propagating in the $E \times B$ direction of the thruster. This oscillation mode is discussed in detail in the next section.

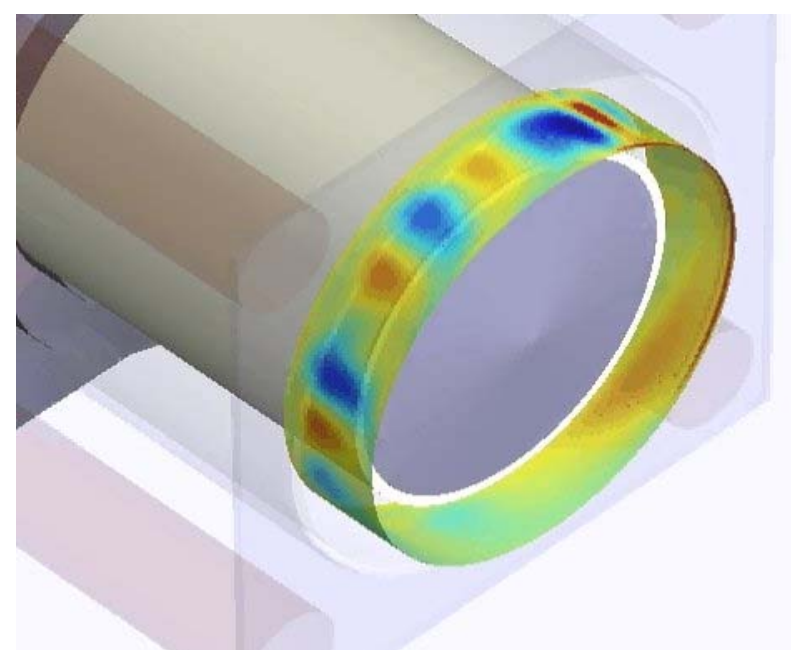

Figure 5. Simulation Visualization: Axial Electron Velocity. 


\section{Results}

The numerical simulation was successful at predicting a number of coherent oscillation modes within the thruster. The most apparent of these was a high frequency oscillation of approximately $74 \mathrm{MHz}$ that occurred in the electron velocity and plasma potential. This oscillation is easily seen if we take a time trace of the electron velocity at a single point centered in the computational domain. This is shown in Fig. 6. However, this data reveals relatively little about the nature and cause of this instability. We can gain further insight into this mode by plotting a streak line in the electron fluid, equivalent to a virtual die injected at a single location in the

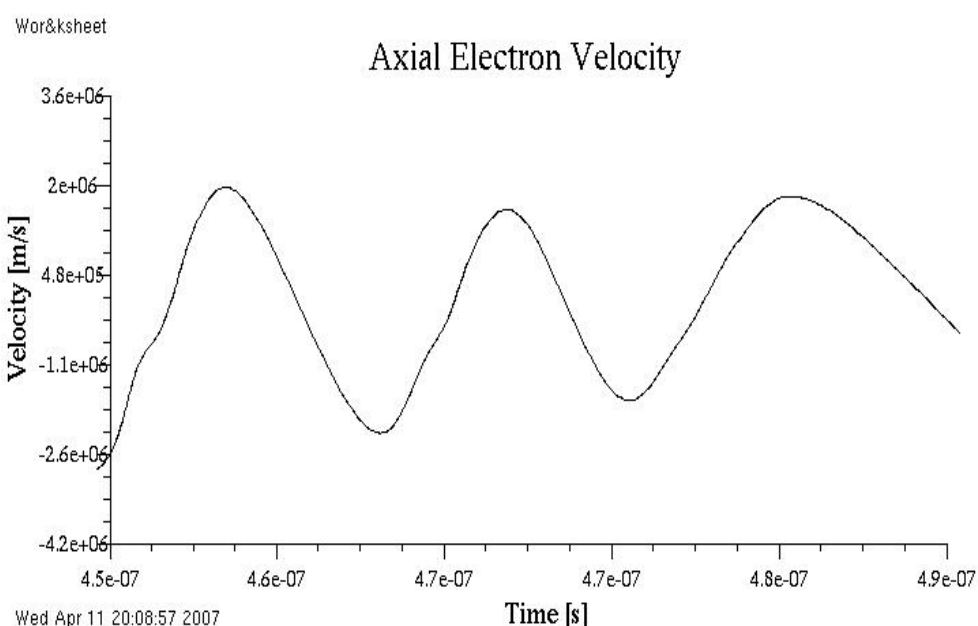

Wed Apr 11 20:08:57 2007 domain. This is shown in Fig. 7. Figure 6. Time Trace of Axial Electron Velocity. Immediately we can observe a structure to this instability that resembles the Kelvin-Helmholtz type instability typically observed in shearing fluid flows. This instability was also predicted on theoretical grounds in an earlier study by Litvak et al. ${ }^{3}$

This high frequency Kelvin-Helmholtz instability is not apparent in the plasma density or ion velocity fields. Instead, a lower frequency oscillation of approximately $7 \mathrm{MHz}$ was observed. This oscillation is clearly visible in a time trace of the plasma density as shown in Fig. 8. This oscillation mode is in good agreement with the experimental measurements of plasma density fluctuations collected by Knoll et al. ${ }^{4}$, and Albarede et al. ${ }^{5}$. The physical mechanism behind these oscillations is not as clear however as with the high frequency electron oscillations discussed above.

It was found that the simulation was able to predict a time-averaged axial electron velocity that was in good agreement with experimental results. This is an encouraging result because no anomalous electron transport term was needed in order to achieve this agreement. It would appear that the plasma oscillations alone provided the necessary contributions to the electron mobility.

Electron Streak - 40ns

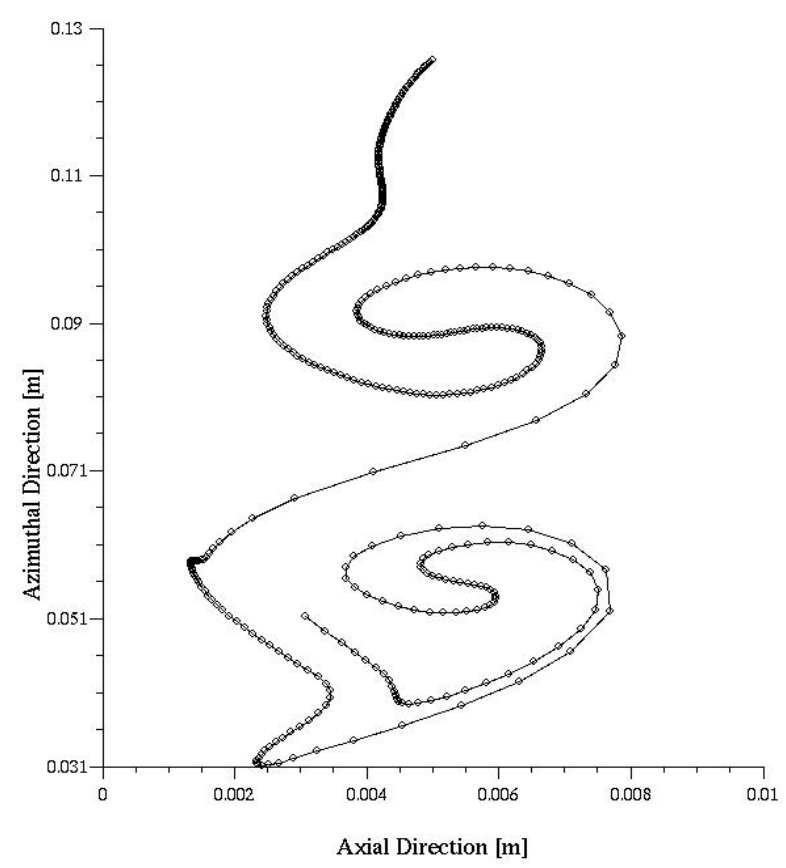

Tue Feb 20 09:09:19 2007

Figure 7. Electron Streak Trace. 


\section{Fluctuating Plasma Density}

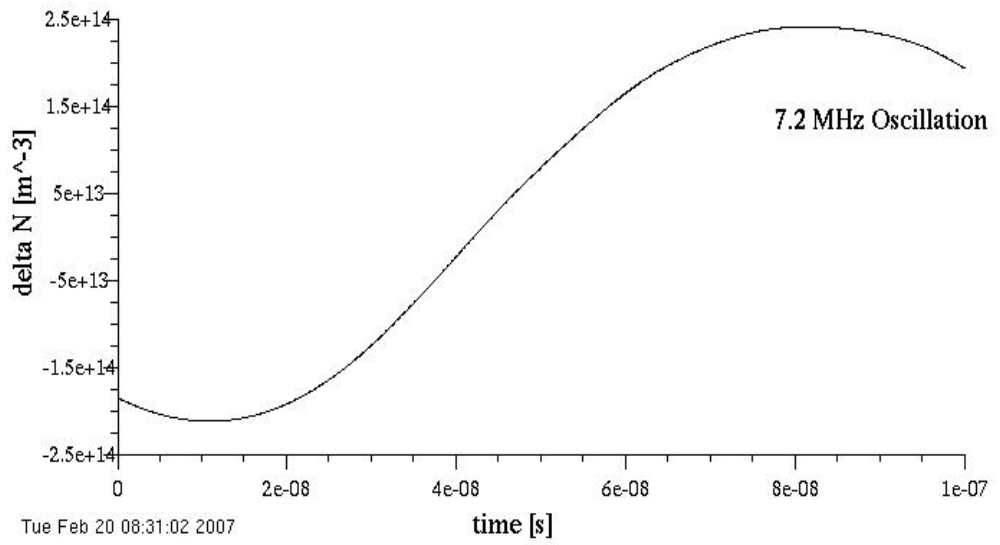

Figure 8. Time Trace of Plasma Density.

In order to compare the experimental and simulated mobilities, we can derive a simple relationship between the time-averaged electron axial and azimuthal drift to the Hall parameter. This is given by

$$
\frac{u_{e z}}{u_{e \theta}}=\frac{1}{\left(\omega_{c e} \tau\right)}
$$

where we neglect spatial variations in density and temperature. We can see from this expression that the axial electron drift scales directly with the inverse Hall parameter. Experimental measurements of the inverse Hall parameter have been collected in work by Meezan et al. ${ }^{1}$. These can be compared to the time-averaged data computed by the simulation. This comparison is made in Fig. 9. We can observe good agreement between experimental and simulated mobility except in the immediate vicinity of the boundaries.

As mentioned previously, several terms were included in the electron momentum equations that are normally neglected in Hall thruster models. By analyzing the raw data produced by the simulation, we can establish which of these terms contributes significantly to the electron transport process in the presence of plasma oscillations. We begin by introducing the classical equation for axial electron velocity without any of these anomalous contributions. This is given by

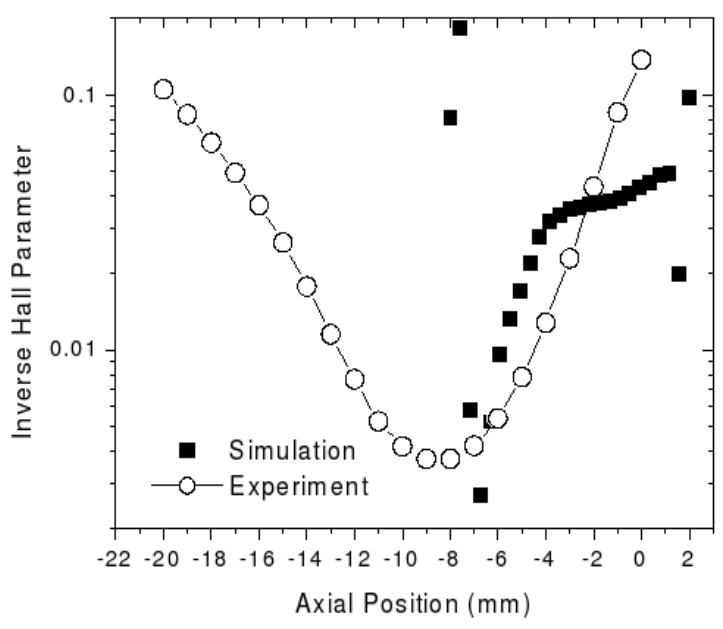

Figure 9. Experimental Versus Simulated Inverse Hall Parameter.

$$
u_{e z}=\frac{-\mu_{e} E_{z}}{1+\left(\omega_{c e} \tau\right)^{2}}-\frac{k}{m_{e} n v_{e}\left(1+\left(\omega_{c e} \tau\right)^{2}\right)} \frac{\partial\left(n T_{e}\right)}{\partial z} .
$$


By solving the axial and azimuthal momentum equations used in this study we can derive a revised expression for the axial electron velocity given by

$$
u_{e z}=\frac{-\mu_{e} E_{z}}{1+\left(\omega_{c e} \tau\right)^{2}}-\frac{k}{m_{e} n v_{e}\left(1+\left(\omega_{c e} \tau\right)^{2}\right)} \frac{\partial\left(n T_{e}\right)}{\partial z}+\psi_{1}+\psi_{2}+\psi_{3}+\psi_{4}+\psi_{5}+\psi_{6}
$$

This equation is essentially the same as the previous, but also includes six additional contributions. The first two additional terms arise from the transient nature of the electron velocity (which is no longer assumed to be constant). They are

$$
\begin{gathered}
\psi_{1}=\frac{-1}{v_{e}\left(\omega_{c e} \tau\right)^{2}}\left(1+\frac{1}{v_{e} R} \frac{\partial u_{e \theta}}{\partial \theta}\right) \frac{\partial u_{e z}}{\partial t} \text {, and } \\
\psi_{2}=\frac{1}{v_{e}\left(\omega_{c e} \tau\right)} \frac{\partial u_{e \theta}}{\partial t} .
\end{gathered}
$$

The third term is related to the azimuthal electric field, which is non-zero in the case of an azimuthal oscillation in the plasma potential. This term becomes

$$
\psi_{3}=\frac{E_{\theta}}{B_{r}}
$$

The fourth term is typically referred to as the diamagnetic drift, and is caused by azimuthal gradients in the plasma density and temperature. This term is given by

$$
\psi_{4}=\frac{k}{m_{e} n v_{e} R\left(\omega_{c e} \tau\right)} \frac{\partial\left(n T_{e}\right)}{\partial \theta} .
$$

The final two terms result from the contribution of the electron axial and azimuthal momentum to the axial velocity. They are

$$
\begin{gathered}
\psi_{5}=\frac{-\mu_{e} E_{z}}{\left(\omega_{c e} \tau\right)^{2}} \frac{1}{v_{e} R} \frac{\partial u_{e \theta}}{\partial \theta}, \text { and } \\
\psi_{6}=\frac{-k}{m_{e} n v_{e}^{2} R\left(\omega_{c e} \tau\right)^{2}} \frac{\partial\left(n T_{e}\right)}{\partial z} \frac{\partial u_{e \theta}}{\partial \theta} .
\end{gathered}
$$

It should be noted that certain simplifications were made in expressing the above six contributions to the electron transport. These simplifications were motivated by the simulation results, and they do not significantly alter the contributions to the transport process. However, in calculating the results below the full expressions for $\psi_{1}$ through $\psi_{6}$ were used.

The contributions from each of the six anomalous terms to the electron mobility was tracked in the simulation results. It was found that the classical transport terms were in fact far smaller than most of the anomalous terms. Four of the six anomalous transport terms were found to dominate the transport process: $\psi_{1}, \psi_{2}, \psi_{3}$, and $\psi_{5}$. The magnitude of these terms has been plotted as a function of time in Fig. 10, for a small representative time window. The average relative contributions of each of these terms to the electron transport has been given below. 


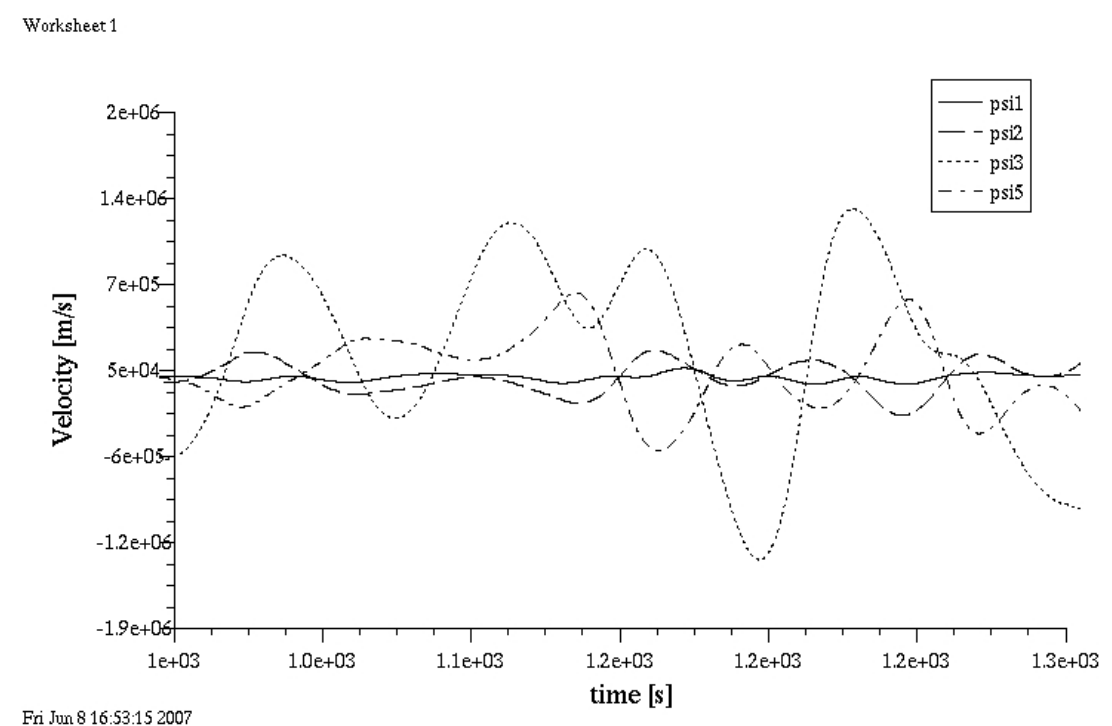

Figure 10. Anomalous Contributions to the Axial Electron Mobility.

We can conclude that the term containing the spatial perturbations in the azimuthal electron velocity $\psi_{5}$ is by far the largest contributer to the overall electron transport process. We can also note that the transient terms $\psi_{1}$ and $\psi_{2}$, as well as the azimuthal field term $\psi_{3}$, play a significant role in establishing the axial electron velocity.

$$
\begin{gathered}
\left\langle u_{e z, \text { classical }}\right\rangle=-9666 \mathrm{~m} / \mathrm{s} \\
\left\langle\psi_{1}\right\rangle=-20795 \mathrm{~m} / \mathrm{s} \\
\left\langle\psi_{2}\right\rangle=-15473 \mathrm{~m} / \mathrm{s} \\
\left\langle\psi_{3}\right\rangle=-24555 \mathrm{~m} / \mathrm{s} \\
\left\langle\psi_{4}\right\rangle=18 \mathrm{~m} / \mathrm{s} \\
\left\langle\psi_{5}\right\rangle=43040 \mathrm{~m} / \mathrm{s} \\
\left\langle\psi_{6}\right\rangle=-5472 \mathrm{~m} / \mathrm{s}
\end{gathered}
$$

\section{Conclusions}

A 2-dimensional simulation of the Hall thruster has been developed in the axial-azimuthal coordinate plane. This model has been successful at predicting various high frequency oscillation modes within the thruster, and modeling the complex interaction between these modes. Two fundamental oscillations were observed in the simulation results. The first was a $74 \mathrm{MHz}$ wave in the electron velocity and plasma potential, which was identified as a Kelvin-Helmholtz type shearing instability. The second was a $7 \mathrm{MHz}$ wave observed in the plasma density and ion velocity fields.

This Hall thruster model was able to self consistently predict the axial transport of electrons in good agreement with experimental results. The contribution of the plasma oscillations to the electron transport process was quantified from the simulation results. The results of this analysis show that the contribution was indeed significant. The most important mechanism driving the axial electron transport was found to be spatial variations in the azimuthal electron velocity. The results of this study suggest that the classical collisional drift of electrons is comparably small in the presence of plasma oscillations in a Hall thruster, and is dominated instead by the presence of these oscillations. 


\section{References}

${ }^{1}$ N. B. Meezan, W. A. Hargus, Jr., and M. A. Cappelli, “Anomalous electron mobility in a coaxial Hall discharge plasma”, Physical Review E., Volume 63, 026410 (2001)

${ }^{2}$ V. I. Baranov, Y. S. Nazarenko, V. A. Petrosov, A. I. Vasin, and Y. M. Yashnov, "Theory of Oscillations and Conductivity for Hall Thruster", 32nd AIAA/ASME/SAE/ASEE Joint Propulsion Conference, Lake Buena Vista, FL, U.S.A., July 1-3, (1996)

${ }^{3}$ A. A. Litvak, N. J. Fisch, "Rayleigh Instabilities in Hall Thrusters", Physics of Plasmas, Vol. 11, No. 4, April (2004)

${ }^{4}$ A. Knoll, C. Thomas, N. Gascon, and M. Cappelli, "Experimental Investigation of High Frequency Plasma Oscillations within Hall Thrusters", 42nd AIAA/ASME/SAE/ASEE Joint Propulsion Conference \& Exhibit, 9 - 12 July (2006)

${ }^{5}$ L. Albarede, S. Mazouffre, A. Bouchoule, and M. Dudeck, "Low-frequency Electron Dynamics in the Near Field of a Hall Effect Thruster", Physics of Plasmas 13, 063505 (2006) 